\title{
The Status and Control Strategy of Bacterial Resistance in China
}

\author{
Xia Zhaofan, Lv Kaiyang, Tang Chenqi, Li Junqiang \\ Department of Burn Surgery, Changhai Hospital, the Second Military Medical University, Shanghai 200433, China
}

\begin{abstract}
Bacterial resistance, a growing public concern worldwide, poses a threat to the health of humans, other animals, and the environment. This paper presents the status of bacterial resistance in various fields in China, analyzes the main causes of such resistance, and reviews the serious damage being caused by it to public health, food safety, the ecological environment, and economic development. We also summarize China's actions to control antimicrobial resistance, and this country's main achievements in this regard. Finally, to address issues in bacterial resistance surveillance, antibiotics stewardship, hazard evaluation, and talent team building, this paper puts forward a national control strategy that calls for the collaborative efforts of multiple disciplines.
\end{abstract}

Keywords: bacterial resistance; antibiotics; sustainable development; control strategy

\section{Introduction}

In recent years, the crisis of bacterial resistance has gradually become severe, and is now a major public safety problem globally that poses a serious threat to the health of humans, animals, and the ecosystem. The prevention and control of bacterial resistance involves many fields, such as medical treatments, agriculture and aquaculture, environmental ecology, as well as many others. The participation of medical personnel, veterinary workers, food and agriculture experts, environmental experts, economists, policymakers, and consumers is required to achieve comprehensive, systematic, and positive results. The sustainable development of society, the economy, and nature will face significant threats if research and cooperation are not conducted in an interdisciplinary, cross-sectoral, and cross-border manner. Further, if we do not effectively control the production and rapid spread of drug-resistant bacteria, it will worsen the threat.

There is no territorial or racial border to the spread of bacterial resistance, and it is difficult for any country to escape from it alone. Therefore, concerted efforts are required to cope with this global crisis. Hence, the World Health Organization issued a global plan of action for the control of bacterial resistance in 2015, appealing to governments to develop national action plans within two years [1], and thus forming a unified global front line for the prevention and control of bacterial resistance. In September 2016, at the G20 (summit of the Group of 20), the issue of antibiotic resistance was raised again on the agenda. The communiqué of the Summit explicitly states: "Antibiotic resistance poses a serious threat to public health, economic growth, and global economic stability." The World Health Organization, the Food and Agriculture Organization of the United Nations, the World Organization for Animal Health, and the Organization for Economic Cooperation and Development were called upon to submit a joint report in 2017 to put forward policy options for addressing this issue and its economic implications [2]. In September 2016, in response to the G20 communiqué, the United Nations General Assembly convened a high-level meeting on antimicrobial drug resistance [3], and such commitments by different nations to work together to combat microbial resistance is a sign that global leaders have recognized the potentially

Received date: January 15, 2017; Revised date: March 13, 2017

Corresponding author: Xia Zhaofan, Department of Burn Surgery, Changhai Hospital, the Second Military Medical University, Professor; Chinese Academy of Engineering, Academician. Major research field is diagnosis and treatment of severe burn and trauma. E-mail: xiazhaofan@163.com

Funding program: CAE Advisory Project “A Study on the Situation and Control Strategy of Drug-Resistant Bacteria and Bacterial Disorder in China" (2015-XY-26)

Chinese version: Xia Zhaofan et al. The Status and Control Strategy of Bacterial Resistance in China. Strategic Study of CAE, https://doi.org/10.15302/ J-SSCAE-2017.02.018 
catastrophic consequences of the bacterial resistance issue. As a large country producing and using antimicrobial drugs, China should undertake its corresponding responsibility and play an important, leading role in solving the global drug resistance crisis.

\section{Drug resistance of pathogenic bacteria from medical and animal sources in China is severe, and drug resistance genes are common in the environment}

Data from the China Bacterial Resistance Monitoring Network (CHINET) showed that the drug resistance of important clinical bacterial isolates to common antimicrobial agents increased gradually in each year from 2005 to 2014. The proportion of multidrug resistance and the incidence of drug-resistant bacteria has increased rapidly, which has posed a serious threat to clinical anti-infection treatments [4-7]. The report of the China Antimicrobial Resistance Surveillance System (CARSS) in 2015 also showed that the detection rate of several important drug-resistant bacteria was still quite high: for example, the detection rate of imipenem-resistant Acinetobacter baumannii $(58.0 \%)$ was remarkably higher in 2015 than that in 2012 (45.8\%). The detection rate of imipenem-resistant Klebsiella pneumoniae (6.8\%) had also noticeably increased in 2015 compared with that in $2014(4.8 \%)$ [8].

China is a country with the most serious problems of drug resistance in animal pathogens [9]. Recently, the resistance rate of Escherichia coli in domestic livestock to ampicillin, doxycycline, and tetracycline has exceeded $90 \%$, and resistance to sulfamide, amoxicillin, streptomycin, and chloramphenicol has been over $50 \%$ [10]. The issue of animal diseases has gradually reached an extreme situation in which there is no longer medicine available for their prevention and control.

The understanding and evaluation of environmental bacterial resistance in China is limited within scientific research. Current studies have shown that a variety of antibiotic resistance genes exist in China. Hundreds of drug resistance genes can be detected in samples collected from farms and their surroundings, some of which express their genotypes at levels tens of thousands of times higher than control samples. This illustrates that the use and disposal of antibiotics generated by human activities are the main sources of antibiotic pollution in natural environments and are facilitating the spread of drug resistance genes. A recent survey demonstrated that multiple antibiotic resistance genes also exist in pathogens isolated from the Beijing smog [11], which indicates that drug resistance genes may spread through different environmental media (even air). Drug resistance genes with the ability to be horizontally transferred can spread among different types of bacteria, thereby increasing the production of drug resistant pathogens, and may enter the human body through the food chain and further endanger human health.

\section{Irrational overuse of antibiotics in the medical and agricultural fields is the direct cause of increasing bacterial resistance}

Bacterial resistance to antibacterial substances is a natural attribute of these microbes. The mechanisms of drug resistance mainly include preventing antibiotics from approaching their targets inside the bacterial cell or genome, changing the antibiotic gene targets, modifying or protecting targets, and modifying the antibiotic directly. Prolonged exposure to antibiotics can cause selective stress on bacteria, and increase drug resistance in their populations.

In China, the use of multiple antibiotics in the medical field is widespread and largely excessive and irrational. Between 2000 and 2010, the use of antibiotics in China increased by $37 \%$, and the total national consumption was only lower than that of India, globally [12]. Data from the Center for Antibacterial Surveillance showed that, in 2014, 63.1533 million daily doses of antimicrobial agents were consumed by inpatients of 167 general hospitals in China. This was worth over 12 billion yuan, but the rate of rational usage of antibiotics was less than $50 \%$. In basic medical and health institutions, less than $1 / 2$ of outpatients and $1 / 4$ of inpatients received reasonable antibiotic treatment, whereas others received irrationally excessive doses [13].

Antibiotics also play an indispensable role in agricultural production, including in animal husbandry, aquaculture products, and the planting industry. However, although it is used to promote growth and prevent infection of animals, the method of using antibiotics in agriculture has aggravated the problem of bacterial resistance. The total use of antibiotics in China in 2013 was about $1.62 \times 10^{5} \mathrm{t}$, of which $52 \%$ was made up of veterinary antibiotics [14], and more than $2 / 3$ of this total amount were used as feed additives. According to conservative estimates, the consumption of antibiotics in animal husbandry in China will be at least double, and constitute nearly $1 / 3$ of the total amount of global consumption, from 2010 to 2030 [15], which will undoubtedly exacerbate the crisis of bacterial resistance and pose a great challenge to regulatory efforts.

The extensive use and disposal of antibiotics by the medical and agricultural fields can therefore lead to retention and transmission of antibiotic residues and drug resistant genes in the environment [16]. In 2013, more than 5 tons of antibiotics were disposed of into the soil and water in China [14]. High concentrations of antibiotics have been detected many times in soil and water samples, especially in the Haihe River Basin in the north and the Pearl River Basin in the south [14,17]. In 2015, an assessment of antibiotic levels in the urine of schoolchildren discovered that Chinese children were generally exposed to lowdose antibiotics; environmental contamination and food may be the main sources of exposure, which shows that the excessive use and disposal of antibiotics can pollute the environment and food, and thus cause potential harm to human health [18]. 


\section{Bacterial resistance will generate widespread harm to food safety, ecological environment, public health, and economic development}

The increasing prevalence of drug-resistant pathogens will seriously affect the healthy operation of aquaculture, endanger food safety, and damage the ecological environment, thereby seriously threatening the health of humans and restricting the sustainable development of global economies.

Studies on antibiotic drug abuse in China showed that infection by drug-resistant bacteria will significantly increase the mortality of patients, prolong hospital stays, and increase medical costs. In 2005, the number of patients who died of antibiotic-resistant infections in China was 489000 , the cost of antimicrobial agents for the treatment of drug-resistant bacteria increased by 3.66 billion yuan, and the cost of hospitalization has risen by 25.2 billion yuan. The direct economic loss caused by antimicrobial drug abuse was estimated to be from 92.55 billion to 98.93 billion yuan, and the indirect economic loss to be 17.37 billion to 18.12 billion yuan. By 2050, bacterial resistance is going to kill 10 million people worldwide, which may exceed the death rate of cancer and make it the leading cause of death among all kinds of human diseases [19].

The report of the World Bank in 2016 stated that if bacterial resistance could not be effectively controlled by 2050 , the cost of global health is likely to increase by 300 billion USD to 1 trillion USD a year, and global livestock production could fall by $7.5 \%$. This would result in economic losses equal to $5 \%$ of the GDP in low-income countries, leaving as many as 28 million people in poverty. The economic damage may even exceed that of the international financial crisis in 2008 [20].

\section{In China, the prevention and control of drug- resistant pathogens has received much attention, and prevention and control efforts have been carried out continuously}

Research on and monitoring of bacterial resistance within the medical field in China began in 1998. Currently, two national antimicrobial resistance surveillance system and a center for antibacterial surveillance have been established. In recent years, the medical field in China has strengthened the management of clinical applications of antimicrobial agents and the development of methods for the prevention and control of drug-resistant pathogens by issuing a series of documents and guidelines, as well as carrying out special activities [21]. Under these conditions, the abuse of antimicrobial drugs in medical institutions has decreased, and the phenomenon of irrational overuse has been controlled. From 2010 to 2014, the use of antimicrobial drugs in inpatients has decreased by $21 \%$ and usage in prescriptions to outpatients has been reduced by
$6 \%$. Moreover, the intensity of antimicrobial use has also declined by $41 \%$ [22].

In July 2015, the Ministry of Agriculture of the People's Republic of China issued the National Plan of Action for the Comprehensive Management of Veterinary Drugs (Antimicrobial Drugs) for the Period 2015-2019. The plan will take five years to further strengthen the supervision of veterinary antimicrobial drugs (including aquatic antimicrobial drugs) and raise the level of scientific and standardized use of veterinary antimicrobial drugs [23]. Five departments, including the Food Safety Office of the State Council of the People's Republic of China, have also started a nationwide campaign to focus on the rectification of antibiotic abuse by the prohibition of compounds and veterinary drug residues in livestock, poultry, and aquatic products. Since 2008, the Ministry of Agriculture of the People's Republic of China has set up an animal-source bacterial resistance monitoring system and launched an annual surveillance plan, which provides important reference materials for risk assessment and control of drug resistance.

In August 2016, fourteen departments, including the National Health and Family Planning Commission and the Development and Reform Commission of the People's Republic of China, jointly issued the National Action Plan for the Control of Bacterial Resistance (2016-2020). It proposed that a working mechanism of joint prevention and control will be established at the national level, and challenges will be met in a multi-sectoral and synergistic manner. This also presents the firm determination and attitude of China towards the fight against bacterial resistance [24].

\section{Prevention and control of bacterial resistance in China should be strengthened in terms of drug resistance monitoring, antibiotic supervision, hazard assessment, and personnel building}

6.1 The surveillance range of bacterial resistance in China is limited, the monitoring ability varies among areas, and the data value is not fully reflected

Currently, drug resistance monitoring in the medical field has not covered basic medical and health institutions, and the level of monitoring of different regions and hospitals varies, which affects the data quality of any large-scale monitoring network. The surveillance of bacterial resistance in animals also has the problem of narrow surveillance coverage and poor representativeness of data. Moreover, there is the phenomenon of "information isolated islands" among the various monitoring networks, accompanied by a lack of data exchange and sharing, thus failing to form "big data" on clinical, animal, food, and environmental drug resistance, and losing any intrinsic interconnections underlying the data. 


\subsection{Supervision and control of antibiotic use and environmental discharge in China still need to be improved}

A large amount of antibiotics is currently used in the medical and agricultural fields in China, some of which are still used in unreasonable amounts. Further improvement, guidance, and supervision are thus needed for long-term regulatory mechanism. Although China has been investigating the concentration of antibiotics in its water for ten years, most of this monitoring is limited to scattered observations and there is a lack of systematic methods and historical comparability. Regarding surveillance and punishment for improper disposal of expired or unqualified antibiotics by pharmaceutical manufacturers and hospitals, there is still a lack of corresponding technical standards and regulatory systems, and a lack of economically applicable techniques for purifying antibiotics in wastewater as well.

\subsection{The hazard assessment of bacterial resistance is insufficient, and further research on drug resistance mechanisms is needed}

At present, there are no published authoritative data in China that can fully reveal the specific harm of bacterial resistance to our public health and economic growth. It is difficult to accurately assess the disease and economic burden caused by drugresistant pathogens, which results in there being a lack of reliable evidence for use in policy making. In addition, knowledge of the molecular epidemiology and mechanisms of bacterial drug resistance should be further strengthened, especially in the fields of epidemic trends, transmission routes, and modes of drug resistance genes in medical institutions, animals, and the environment. The mechanisms of bacterial resistance should be further elucidated to provide scientific support for the formulation of prevention and control strategies.

\subsection{There is urgent need of qualified personnel for prevention and control of bacterial resistance}

Currently, there is a shortage of unified guidance for the prevention and control of bacterial resistance in various fields. Interdisciplinary and cross-sectoral expert advisory teams in the fields of medical health, agriculture, environment, food, pharmaceuticals, economics, and education, are needed and are insufficient. It is not possible to provide policy makers and the public with professional advice from macro-policy formulation to practical monitoring. Moreover, the setting and staffing of infection management departments in some medical institutions are not enough to meet the requirements for carrying out the required work properly. Professional teams with permanent members are very few in number, and the development of professional disciplines is restricted. Similar problems of talent echelon construc- tion are seen in the fields of animal husbandry and environmental monitoring, but need to be resolved.

\section{Recommendations for the development of a strategy for prevention and control of bacterial resistance with multi-field cooperation and joint departments}

7.1 Set up a working group or committee for the prevention and control of bacterial resistance, and develop a mediumand long-term strategic plan

Prevention and control of drug-resistant pathogens is a systematic engineering problem, which requires the set-up of a special working group or committee at the national strategic level, based on knowledge of national conditions, to carry out a medium- and long-term strategic plan, and provide guidance in and promotion of prevention and control work. In particular, cooperation mechanisms should be established in the fields of medical treatment, agriculture, and environmental studies, as well as food and drug regulatory departments, to coordinate human, material, and financial resources within the various departments. The national action plans issued at this time require that the advantages of joint prevention and control be taken through the national level of comprehensive prevention and control, to realize the whole process of supervision of antimicrobial agents and face the challenge of drug-resistant bacteria in a coordinated manner [24].

\subsection{Strengthen monitoring and management of bacterial resistance and the use of antimicrobial agents in each field}

Comprehensive and systematic monitoring is the cornerstone of prevention and control. We should rely on existing resources and integrate the advantages of all parties to establish transparent and open monitoring networks of bacterial resistance and usage of antimicrobial agents. Strengthening the cooperation among each department, establishing mechanisms for connection among departments, and realizing the sharing and exchange of information among them, will be needed to provide support for usage and management of antimicrobial drugs in the fields of medicine and agriculture. The National Antimicrobial Resistance Monitoring System for Enteric Bacteria (NARMS) of the United States can be taken as an example of this, as it is an inter-departmenta collaborative project established by the United States Centers for Disease Control, Food and Drug Administration, and Department of Agriculture. This system monitored drug resistance of enterogenic bacteria in humans, retail meat, and edible animals at the same time. It is also important for such a collaborative effort to integrate and analyze their data, as well as compile and publish reports regularly. This establishes a foundation for the prevention and control of the spread of bacterial resistance in 
different fields, and provides the support for intervention measures as well.

On the other hand, we should continue to enhance the management of antimicrobial agents and the monitoring of antibiotic disposal, improve the relevant regulations and management system for the usage of antimicrobial drugs, and strictly implement the process. Classification management of veterinary drugs and gradually banning antibiotics are ways to promote the growth of animals without worsening the bacterial resistance problem. Rectifying and integrating enterprises of producing antimicrobial drugs, strict management of marketing, and promotion of industrial upgrades are needed. It will also be important to strengthen the ability of environmental monitoring and law enforcement of antibiotics, so that we can better grasp the origin, distribution, historical evolutionary trends, environmental persistence, and transformation mechanisms of typical antibiotics in the natural environment of China.

\subsection{Strengthen basic and applied research on bacterial resistance}

Through policy guidance and mechanism construction, we could increase research and investment in the control of multidrug-resistant bacterial infections and the management of antimicrobial agents. In terms of basic research, it is necessary to elucidate the generation and transmission mechanisms of drug resistance, master the distribution and prevalence of drugresistant bacteria and drug-resistant genes, and determine the key factors that affect the emergence and transmission of drug resistance. In terms of applied research, we should pay attention to the research and development of new antimicrobial agents, rapid diagnosis technology, antibiotic substitutes and vaccines, and explore technologies for treatment and removing antibiotic contamination from the environment. Active research on the health and economic burden of drug-resistant pathogens should be conducted to provide reliable evidence for the formulation and implementation of related policies.

\subsection{Strengthen the construction of talent teams for} prevention and control, and perform well in publicizing the issue and educating the public

The implementation of various prevention and control efforts needs to be guaranteed by a professional team. It is still necessary to further optimize the echelon construction of talented teams in various fields in the future. We should set up a multi-disciplinary management team for drug-resistant pathogens, enhance the ability of professionals to prevent and control these, and improve the level of professional management. In order to ensure prevention and control of bacterial resistance, China will set up a cross-disciplinary advisory expert committee to provide advice and policy recommendations for management and control of antimicrobial agents [24].
Additionally, arousing awareness of prevention and control in the public and relevant practitioners can play an important role in the effective implementation of national policies. We should strengthen the education and training of medical and animal breeding practitioners, improve public awareness about the harm caused by bacterial resistance, and promote rational drug use.

\subsection{Strengthen international cooperation and exchanges for prevention and control of bacterial resistance}

Bacterial resistance is a public health problem that we need to face together. To effectively curb the spread of bacterial resistance over the world has been a goal highly valued by governments and international organizations. China should strengthen its cooperation and exchange with developed countries and international organizations, promote sharing in our efforts toward bacterial resistance monitoring and risk assessment, antimicrobial drug management, prevention and control strategy formulation, and research and development of new technology products. This would play a positive and important role in the battle against the crisis of bacterial resistance, as well as enhance the international image of China.

\section{References}

[1] World Health Organization. Global action plan on antimicrobial resistance [R]. Geneva: WHO, 2015.

[2] G20 Leaders' Communique Hangzhou summit [J]. China Eco-nomic Weekly, 2016 (36): 98-105. Chinese.

[3] World Health Organization. United Nations high-level meeting on antimicrobial resistance [EB/OL]. (2016-08-05) [2016-11-21]. http://www.who.int/mediacentre/events/2016/antimicrobial-resis-tance/zh/. Chinese.

[4] Hu F P, Zhu D M, Wang F, et al. CHINET 2014 surveillance of bacterial resistance in China [J]. Chinese Journal of Infection and Chemotherapy, 2015, 15(5): 401-410. Chinese.

[5] Tian L, Chen Z J, Sun Z Y, et al. Antibiotic resistance profile of enterobacter in hospitals across China: Data from CHINET anti-microbial resistance surveillance program from 2005 through 2014Chinese Journal of Infection and Chemotherapy, 2016, 16(3): 275-283. Chinese.

[6] Zhang H, Zhang X J, Xv Y C, et al. Resistance profile of acinetobacter isolates in hospitals across China: Results from CHINET antimicrobial resistance surveillance program 2005-2014 Chinese Journal of Infection and Chemotherapy, 2016, 16(4): 429-436. Chinese.

[7] Xv A, Zhuo C, Su D H, et al. Changing susceptibility of klebsiella strains in hospitals across China: Data from the CHINET antimi-crobial resistance surveillance program 2005-2014 [J]. Chinese Journal of Infection and Chemotherapy, 2016, 16(3): 267-274. Chinese.

[8] Committee of Experts on Rational Drug Use, National Health and Family Planning Commission of the P.R.China, and China Anti-microbial Resistance Surveillance System. China antimicrobial 
resistance surveillance system report 2015 [J]. China Licensed Pharmacist, 2016, 13(3): 3-8. Chinese.

[9] Shen J Z. The risk and prevention of antimicrobial resistance among pathogenic bacteria from food animals. Proceedings of the National Health Technology Forum [C]. Yantai: China National Center for Biotechnology Development, 2009. Chinese.

[10] Jiang H, Lv D, Chen Z, et al. High prevalence and widespread distribution of multi-resistant escherichia coli isolates in pigs and poultry in China [J]. The Veterinary Journal, 2011, 187(1): 99-103.

[11] Pal C, Bengtsson-Palme J, Kristiansson E, et al. The structure and diversity of human, animal and environmental resistances $[\mathrm{J}]$. Mi-crobiome, 2016, 4(1): 54.

[12] Van Boeckel T P, Gandra S, Ashok A, et al. Global antibiotic con-sumption 2000 to 2010: An analysis of national pharmaceutical sales data [J]. The Lancet Infectious Diseases, 2014, 14(8): $742-750$.

[13] Wang J, Wang P, Wang X, et al. Use and prescription of antibiotics in primary health care settings in China [J]. JAMA Internal Medi-cine, 2014, 174(12): 1914-1920.

[14] Zhang Q, Ying G, Pan C, et al. Comprehensive evaluation of anti-biotics emission and fate in the river basins of China: Source anal-ysis, multimedia modeling, and linkage to bacterial resistance [J]. Environmental Science \& Technology, 2015, 49(11): 67726782.

[15] Van Boeckel T P, Brower C, Gilbert M, et al. Global trends in antimicrobial use in food animals [J]. Proceedings of the National Academy of Sciences, 2015, 112(18): 5649-5654.

[16] Finley R L, Collignon P, Larsson D G J, et al. The scourge of antibiotic resistance: The important role of the environment [J]. Clinical Infectious Diseases, 2013, 57(5): 704-710.

[17] Xiong W, Sun Y, Zhang T, et al. Antibiotics, antibiotic resistance genes, and bacterial community composition in fresh water aqua- culture environment in China [J]. Microbial Ecology, 2015, 70(2): $425-432$

[18] Wang H X, Wang B, Zhao Q, et al. Antibiotic body burden of Chinese school children: A multisite biomonitoring-based study $[\mathrm{J}]$ Environmental Science \& Technology, 2015, 49(8): 5070-5079.

[19] O'Neill J. Review on antimicrobial resistance - antimicrobial resistance: Tackling a crisis for the health and wealth of nations [EB/ OL]. (2014-12-16) [2016-10-25]. http://www.west-info.eu/drugresistant-infections-could-lead-to-10-million-extra-deaths-a-year/ amr-review-paper-tackling-a-crisis-for-the-health-and-wealth-ofnations_1/.

[20] World Bank. Drug-resistant infections: A threat to our economic future (discussion draft) [EB/OL]. (2016-03-20) [2016-10-25]. http://www.worldbank.org/en/topic/health/publication/drug-resistant-infections-a-threat-to-our-economic-future 2016.

[21] Xiao Y, Zhang J, Zheng B, et al. Changes in Chinese policies to promote the rational use of antibiotics [J]. PLoS Medicine, 2013, 10(11): e1001556.

[22] National Health and Family Planning Commission of the PRC. Antimicrobial management will be enhanced in multi areas [EB/ OL]. (2015-11-19) [2016-11-22]. http://www.nhfpc.gov.cn/xcs/ wzbd/201511/5f5da3a6792c479aa91cd05e2328d836.shtm1. Chinese.

[23] Ministry of Agriculture of the PRC. Five year action plan for the comprehensive management of veterinary drugs in China (20152019) [EB/OL]. (2015-07-23) [2016-11-23]. http://www.moa.gov. cn/govpublic/ncpzlaq/201507/t20150723_4759838.htm. Chinese.

[24] National Health and Family Planning Commission of the PRC. National action plan for contain antimicrobial resistance (20162020) [EB/OL]. (2016-08-25) [2016-11-23]. http://www.moh.gov. cn/yzygj/s3593/201608/f1ed26a0c8774e1c8fc89dd481ec84d7 shtml. Chinese. 